\title{
Research Paper \\ Effects of 5A Model-based Intervention on Self-care and Quality of Life in Elderly People With Hypertension
}

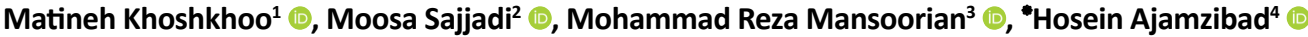

1. Department of Geriatric Nursing, Social Development and Health Promotion Research Center, School of Nursing, Gonabad University of Medical Sciences, Gonabad, Iran.

2. Department of Internal Surgery Nursing, Social Development and Health Promotion Research Center, School of Nursing, Gonabad University of Medical Sciences, Gonabad, Iran.

3. Department of Community Health Nursing and Nursing Management, Social Development and Health Promotion Research Center, School of Nursing, Gonabad University of Medical Sciences, Gonabad, Iran.

4. Department of Nursing and Elderly Health, Nursing Research Center, School of Nursing, Gonabad University of Medical Sciences, Gonabad, Iran.

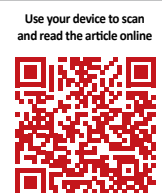

Citation: Khoshkhoo M, Sajjadi M, Mansoorian MR, Ajamzibad H. [Effects of 5A Model-based Intervention on Self-care and Quality of Life in Elderly People With Hypertension (Persian)]. Iranian Journal of Ageing. 2021; 16(3):348-361. http://dx.doi.org/10.32598/ sija.2021.16.3.657.1

http://dx.doi.org/10.32598/sija.2021.16.3.657.1

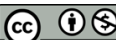

Received: 10 Jan 2021

Accepted: 24 Apr 2021

Available Online: 01 Oct 2021

Keywords:

Elderly, 5A model,

Self-care, Quality of

Life, Hypertension

\section{A B S T R A C T}

Objectives Hypertension is one of the most common chronic diseases in old age. The self-care and quality of life of older people can improved by appropriate interventions. This study aims to assess the effects of an intervention based on 5A model on self-care and quality of life in the elderly with hypertension. Methods \& Materials This quasi-experimental study was conducted on 80 older people with hypertension. They were randomly assigned into two groups of intervention and control. The intervention group received the $5 \mathrm{~A}$ model-based treatment for two months, while the control group received only the routine care from community health centers. Before and after the intervention, data were collected using a demographic form, behavior subscale of the Hypertension Self-Care Profile questionnaire, and the Control, Autonomy, Self-Realization and Pleasure (CASP-19) questionnaire. The collected data were analyzed using independent t-test, paired t-test, Mann-Whitney U test, Chi-square test, and Fisher's exact test in SPSS v. 20 software.

Results The Mean $\pm S D$ self-care scores of the intervention group significantly increased $(P<0.001)$ after the intervention $(50.95 \pm 4.77)$ compared to pre-intervention score $(46.67 \pm 5.83)$ and compared to the control group $(40.92 \pm 4.86)$. The Mean $\pm S D$ quality of life scores of the intervention group also significantly increased $(P<0.001)$ after the intervention $(39.02 \pm 6.76)$ compared to pre-intervention score $(33.65 \pm 8.03)$ and compared to the control group (24.97 \pm 7.19$)$.

Conclusion The intervention based on $5 \mathrm{~A}$ model can improve self-care and quality of life of the elderly. This intervention, as a fast, accessible and easy method can be used in the health system to improve the health of the elderly.

\section{* Corresponding Author:

Address: Department of Nursing and Aging, Faculty of Nursing College, Nursing Research Center, Gonabad University of Medical Science, Gonabad, Iran. Tel: +98 (515) 7223028

E-mail: ajam.h@gmu.ac.ir 


\section{Extended Abstract}

\section{Introduction}

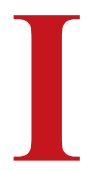

ncreased life expectancy and decreased fertility rate in Iran has led to an increase in the number of older people [1]. Hypertension is one of the most common chronic diseases in the elderly that plays an important role in their quality of life [2]. Blood pressure control requires self-care and patient involvment in self-care [3, 4]. Selfcare guidelines can play an important role in regulating and controlling blood pressure, but most patients do not follow the instructions $[5,6]$. With proper intervention, self-care and quality of life in the elderly with hypertension can be improved. The 5A model for self-management is a behavior change model that is designed based on an evidencebased approach and is suitable for managing health and creating desirable behaviors, including self-care behaviors [7]. This study aims to assess the effect of a 5A model-based intervention program on self-care and quality of life in the elderly with hypertension.

\section{Methods}

This is a quasi-experimental study with an ethical approval obtained from the Ethics Committee of Gonabad University of Medical Sciences (Code: IR.GMU.REC.1399.044). Participants were 80 older people randomly assigned into two groups of intervention $(n=40)$ and control $(n=40)$ groups. The sample size was estimated 36 for each group according to similar study [8], and increased to 40 due to the possibility of sample drop. Inclusion criteria were: willingness to participate in the study, age 60-75 years, having hypertension, receiving at least one blood pressure medication, no cognitive impairment, speech or hearing problems, and no serious medical condition. Exclusion criteria were: Absent from a training session or not answering the telephone call, having an accident, death during an intervention, and change in medication regimen.

For data collection, a demographic form, recording of blood pressure, behavior subscale of the Hypertension SelfCare Profile (HSCP) questionnaire, the Control, Autonomy, Self-Realization and Pleasure (CASP-19) questionnaire, and a desk mercury sphygmomanometer (Riester Diplomat 1002 model) were used. The Persian version of HSCP was validated $(\alpha=0.85)$ for Iranian elderly by Barati et al. [33], and the Persian version of CASP-19 was validated ( $\alpha$ $=0.80$ ) by Heravi et al. [34]. Blood pressure was measured from the patient's right arm in a sitting position [11].
Questionnaires were first completed before the intervention. Educational intervention based on the $5 \mathrm{~A}$ model was performed for 2 months. The educational content included self-care and dimensions of quality of life for the elderly. The intervention was performed in one session via faceto-face (during five stages of Ask, Advise, Assess, Assist, and Arrange) and in 1-4 sessions via telephone calls. The control group received routine care from community health centers for two months. At the end of the intervention, questionnaires were completed again by two groups. Data were analyzed by Shapiro-Wilk test, independent t-test, MannWhitney U test, paired t-test, Chi-square test, and Fisher's exact test.

\section{Results}

Participants were 80 older people with a Mean \pm SD age of $64.58 \pm 3.47$ years. Most of them were male (51.3\%), married (91.3\%) and housekeeper (42.5\%). The mean duration of hypertension in them was $14.01 \pm 6.92$ years. The two groups were homogeneous in terms of demographic characteristics including age, body mass index, hours of activity (per month), hours of sleep (per day), disease duration (month), marital status, level of education, economic status, employment status, and diet.

The Mean \pm SD pre-test self-care score of the intervention group (46.67 \pm 5.83$)$ was significantly different from that of the control group $(43.75 \pm 5.46)(\mathrm{P}=0.023)$. The mean posttest self-care scores of the intervention $(50.95 \pm 4.77)$ and control (40.92 \pm 4.86$)$ groups was also significantly different $(\mathrm{P}<0.001)$. According to the results of paired t-test in Table 1 , there was a statistically significant difference between the mean pre-test and post-test self-care scores of the intervention group $(\mathrm{P}<0.001)$, and between pre-test and post-test self-care scores of the control group $(\mathrm{P}<0.001)$.

The Mean \pm SD pre-test quality of life score of the intervention group (33.65 \pm 8.03$)$ was significantly different from that of the control group $(25.55 \pm 8.32)(\mathrm{P}<0.001)$. The mean post-test quality of life score of the intervention group (39.02 \pm 6.76$)$ and the control group (24.97 \pm 7.19$)$ was also significantly different $(\mathrm{P}<0.001)$. According to the results of paired t-test in Table 1, there was a statistically significant difference between the mean pre-test and post-test quality of life scores of the intervention group $(\mathrm{P}<0.001)$, but no significant difference was found in the control group $(\mathrm{P}=0.352)$.

\section{Discussion and Conclusion}

Intervention based on $5 \mathrm{~A}$ model for 8 weeks affects the self-care and quality of life of the elderly with hypertension. It seems that using this technique in the health system 
Table 1. Difference in the means of self-care and quality of life before and after the intervention in the two groups

\begin{tabular}{cccc}
\hline Variables & \multicolumn{2}{c}{ Mean \pm SD } & Sig.* \\
\cline { 2 - 3 } Mean difference in self-care & Intervention Group & Control Group & $<0.001$ \\
Mean difference in quality of life & $4.27 \pm 3.27$ & $-2.82 \pm 2.33$ & $<0.001<$ \\
\hline
\end{tabular}

*Independent t-test.

as a fast, accessible and easy intervention can be effective in improving the health status of the elderly. Further studies on the elderly with other chronic diseases can be effective in using this model as part of the geriatric nursing care program in Iran.

\section{Ethical Considerations}

Compliance with ethical guidelines

This study was approved by the Ethics Committee of Gonabad University of Medical Sciences (Code: IR.GMU. REC.1399.044). All ethical principles are considered in this article. The goals and procedure of the research was explained to the patients. The written informed consent form was taken from them. The patients were free to leave the study at any time.

Funding

This study was extracted from a MA. thesis of the first author at the Department of Geriatric Nursing, Social Development and Health Promotion Research Center, School of Nursing, Gonabad University of Medical Sciences, Gonabad.

\section{Authors' contributions}

Conceptualization, methodology, editing, final writing, research: All authors; Analysis: Moosa Sajjadi; Drafting: Matineh Khoshkhoo and Hosein Ajamzibad; Supervision: Hosein Ajamzibad, Moosa Sajjadi and Mohammad Reza Mansoorian; Project management: Hosein Ajamzibad.

\section{Conflicts of interest}

The authors declare no conflict of interest

\section{Acknowledgements}

The authors would like to thank the Vice-Chancellor for Education and Research of Gonabad University of Medical Science, Community Health Centers In Gonabad, and the elderly participated in the study for their support and cooperation. 


\section{مقاله يخوهشى \\ ثأثير آزمايش مبتنى بر مدل 5 بر خودمراقبتى و كيفيت زندكَى سالمندان مبتالا به يرفشارى خون

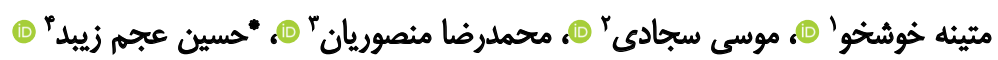

ا.كروه يرستارى سالمندى، مركز تحقيقات توسعه اجتماعى وارتقاى سلامت، دانشكده يرستارى، دانشعاه علوميزشكى كناباد، كناباد، ايران.

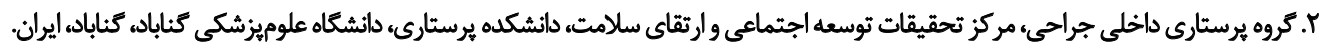

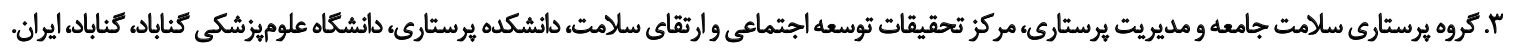

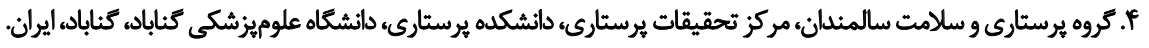

\section{حكיد}

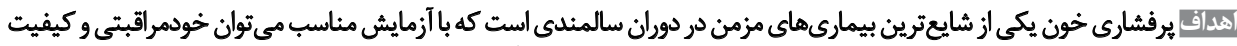

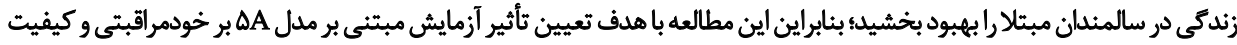

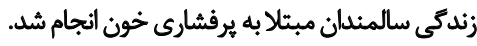

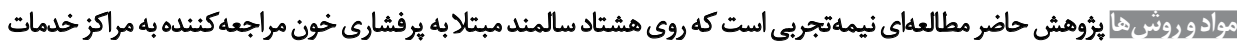

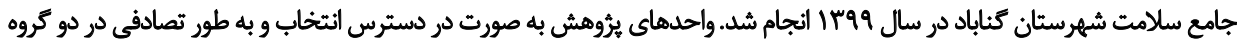

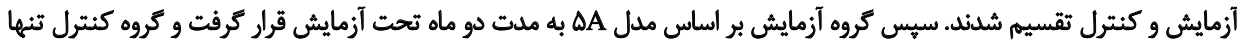

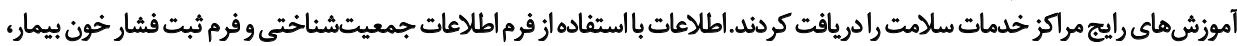

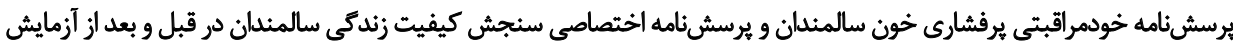

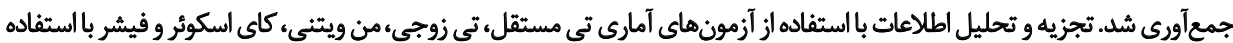

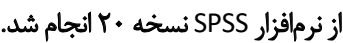

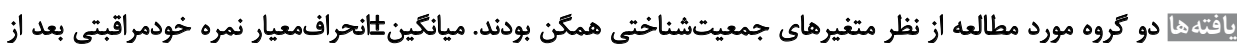

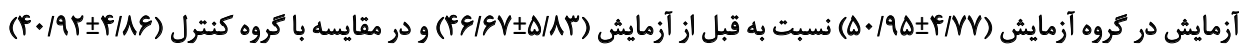

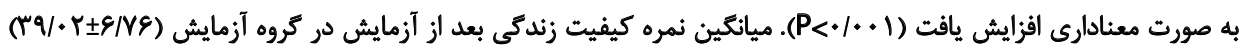

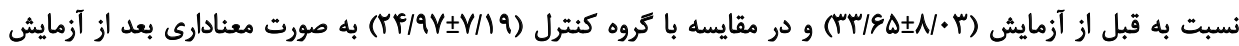

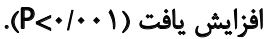

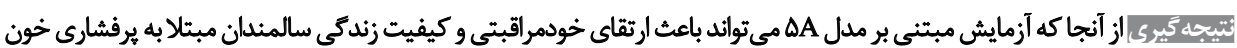

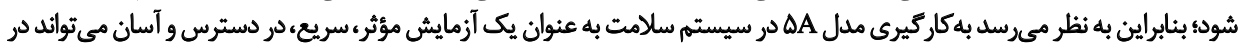

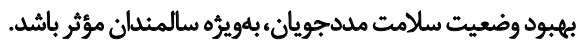

تاريخ دريافت: الrدى دوبا

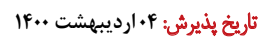

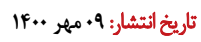

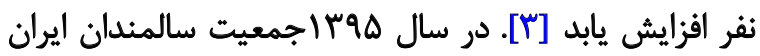

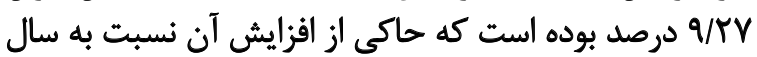

dotes

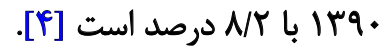

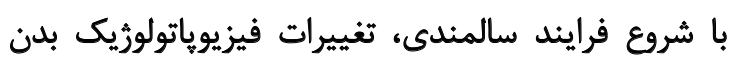

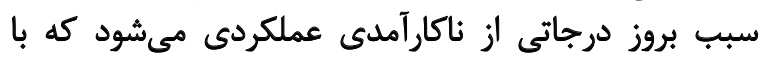

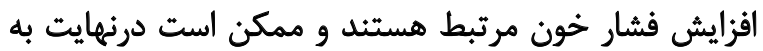

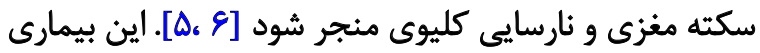

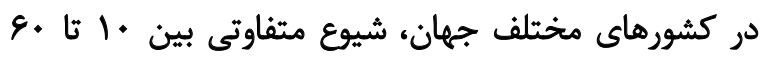

إبهبود شرايط زندگى و مراقبتهاى بهداشتى درمانى سبب

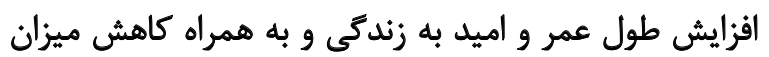

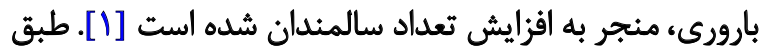

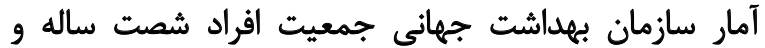

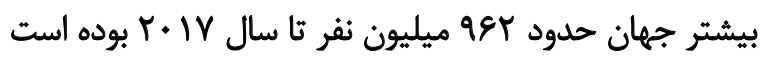

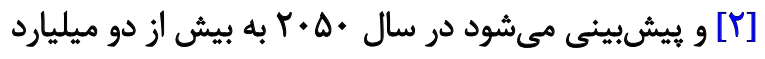




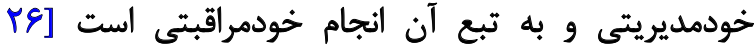

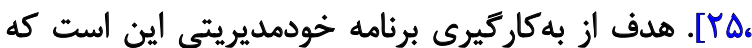

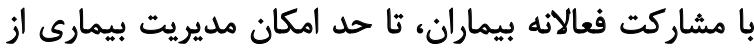

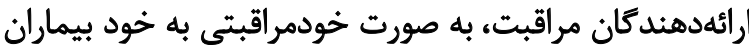

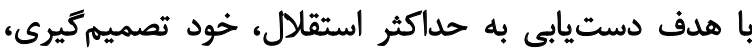

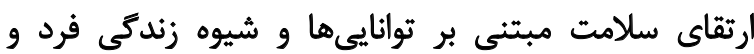
همجنين افزايش كيفيت زندكى منتقل شود [Tr]

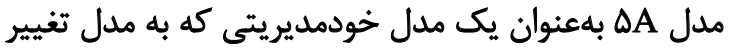

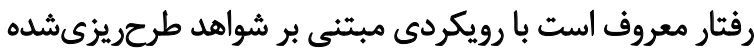

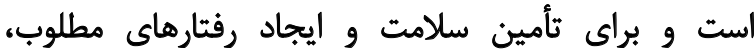

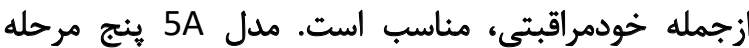

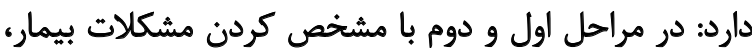

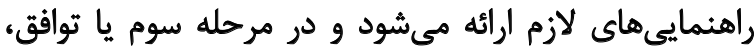

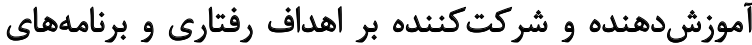

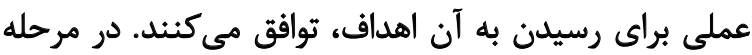

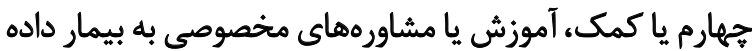

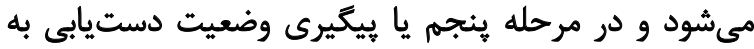

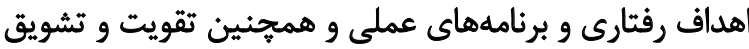

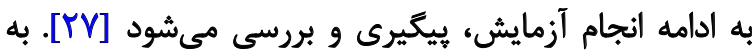

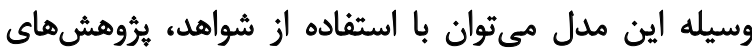

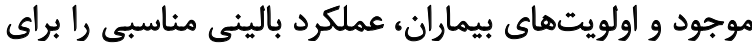

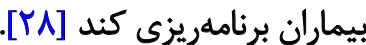

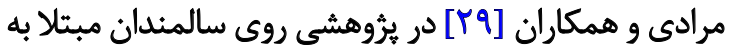

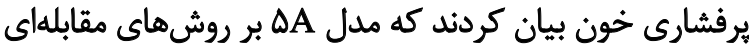

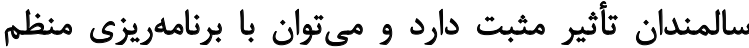

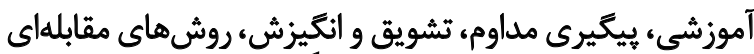
مناسب را در بيماران مزمن، خصوصا سالمندان ارتقا داد.

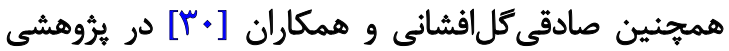

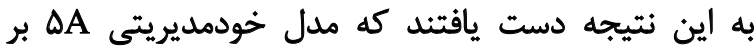
خودكارآمدى بيماران سالمند ديابتى اثر دارد و واين اين روشي

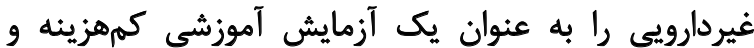

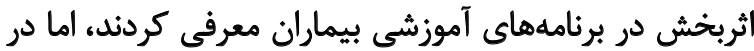

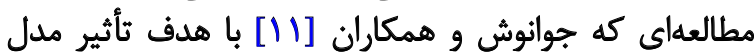
خودمديريتى 5A بر كيفيت زندكى سالمئي

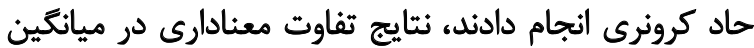

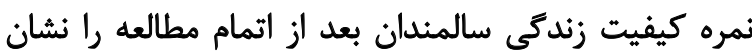

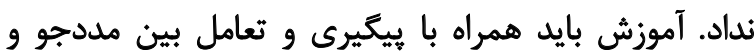

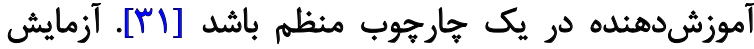

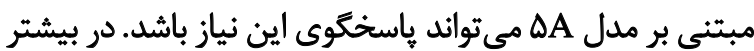

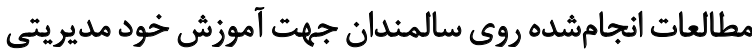

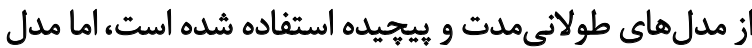

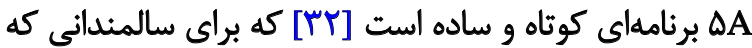

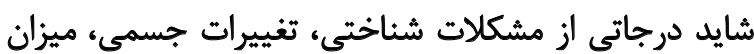

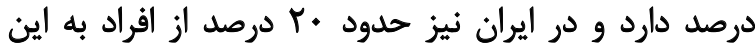

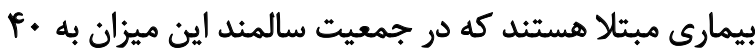
درصد افزايش يافته است [V]

فشار خون بالا در افراد سالمند، علاوه بر بُعد جسمانى در

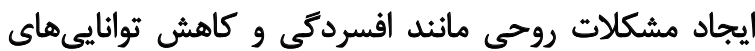

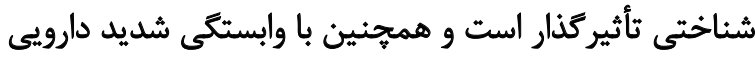

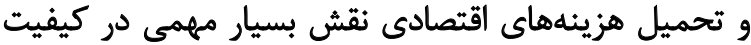
زندكى سالمندان ايفا مي كند [A]].

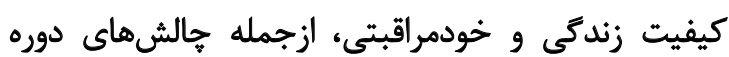

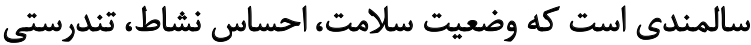

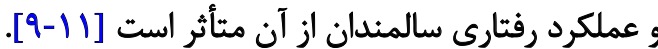
كنترل فشار خون نيازمند خودمراقبتى است و مشاركت

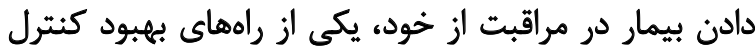

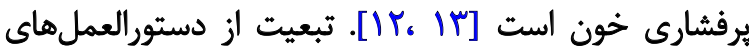

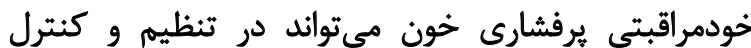

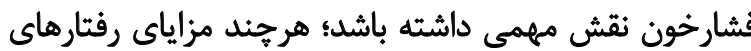

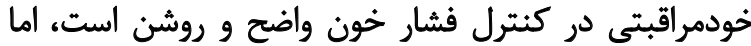

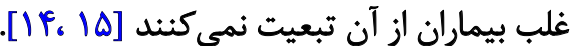
در سالمندان خودمراقبتى باعث ميشود كه ادارهكنتده

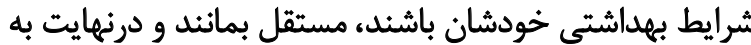

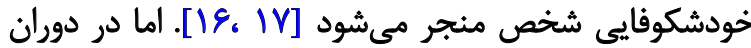

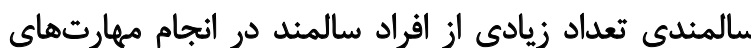

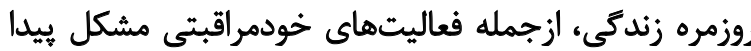

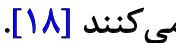

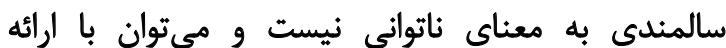

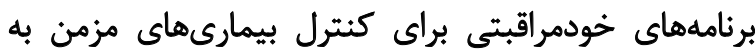

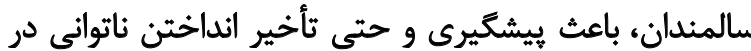

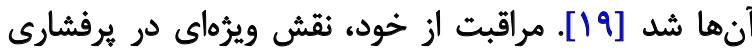

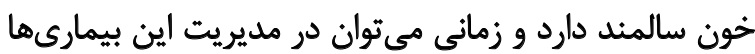

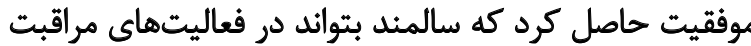

از خود نقش فعالى داشته باشد [•r]

سالمندان به علت داشتن شرايط خاص، بيمارىهاي مزٔمن،

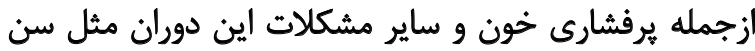

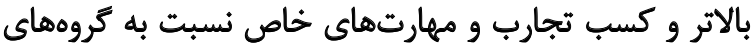

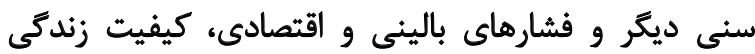

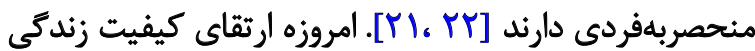

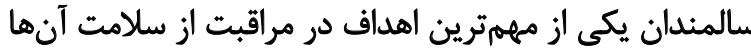

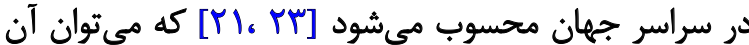

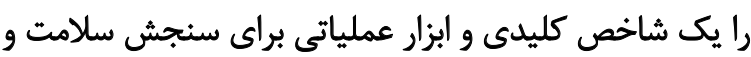

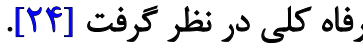
يكى از روشهاى مديريت بيمارى توسط فرد، بهكارگيرى 


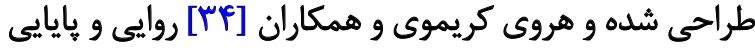

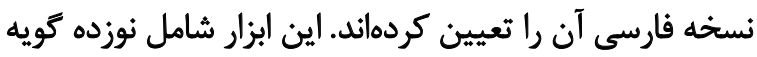
در هجار بُعد كنترل، استقلال، خوديابي و كاميابي است.

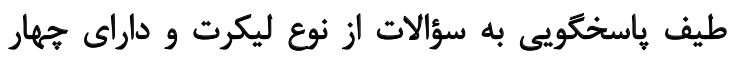

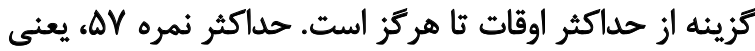

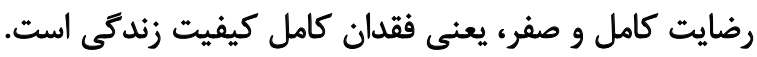

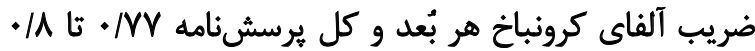

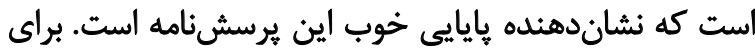

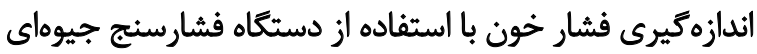

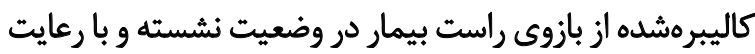

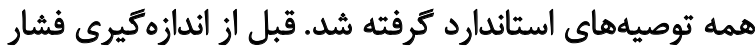

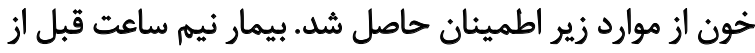

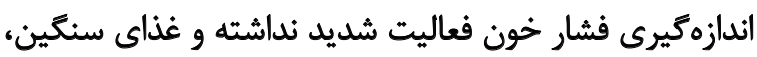

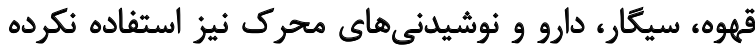

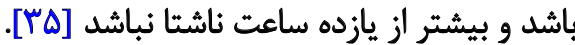

يرسش نامهها قبل از آزمايش توسط افراد هر دو تروه تكميل

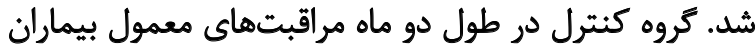

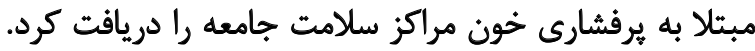

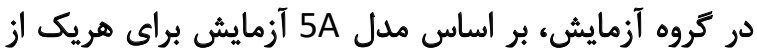

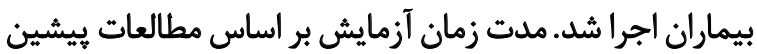

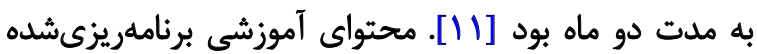

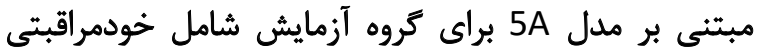

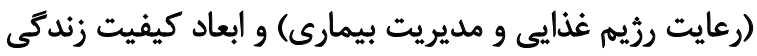

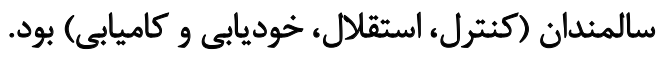

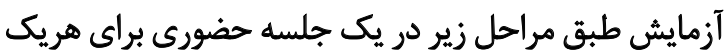

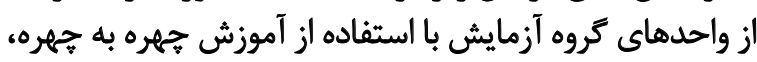

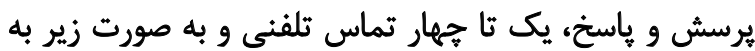

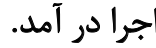

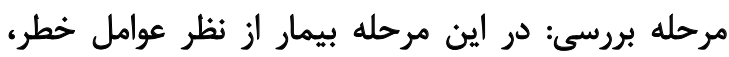

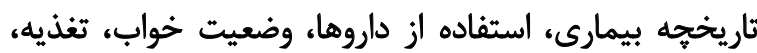

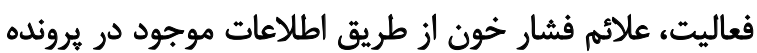

و مصاحبه حضورى بررسى شدار خون ازطريق

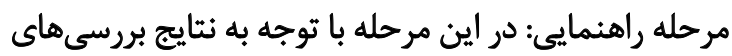

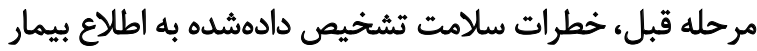

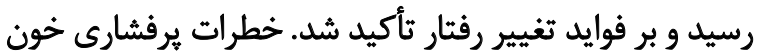

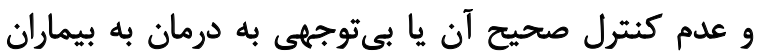

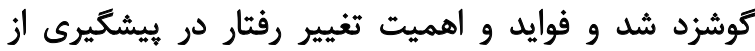
عوارض بيمارى توضيح داده شد.

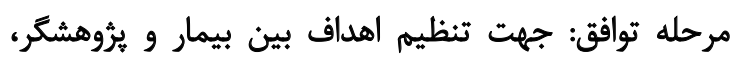

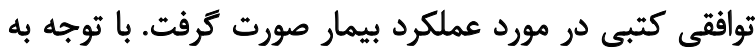

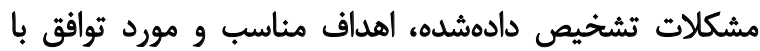
بيمار تعيين و براى هريك از اهداف، برنامه عملى طرح إديزى شد.
تحمل هايين براى يادكيرى طولاتىمدت و همجنين حجم

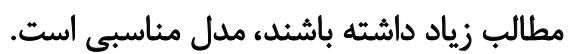

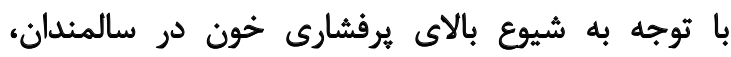

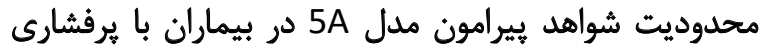

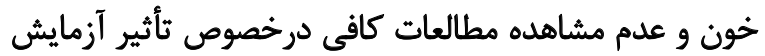

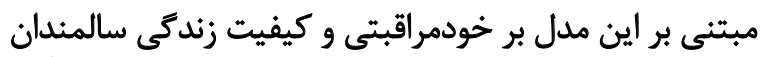

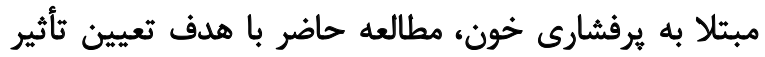

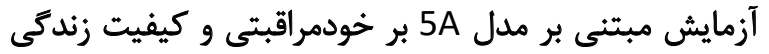

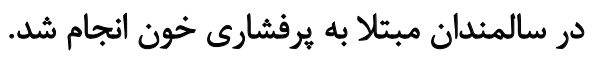

ورش مطالعه

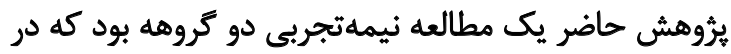

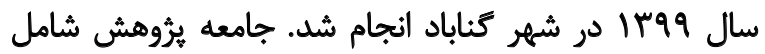

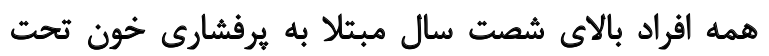

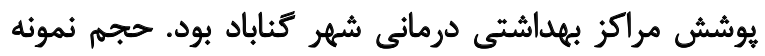

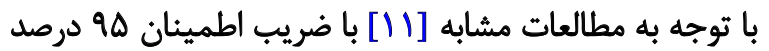

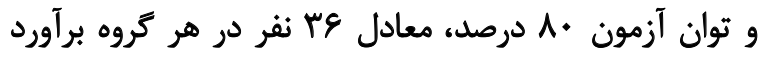

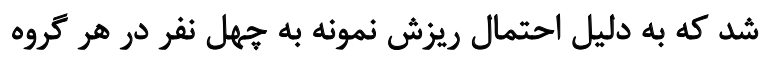

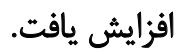

نمونه كيرى به صورت دسترس از مراكز سلامت جامعه شهر

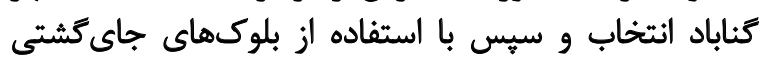

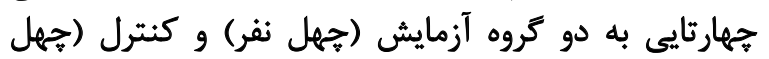

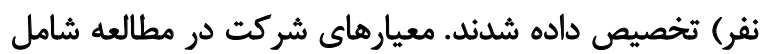

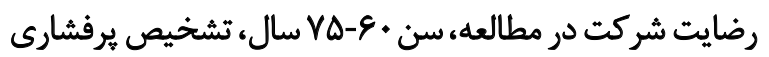

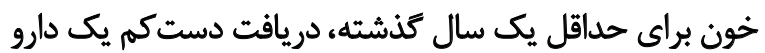

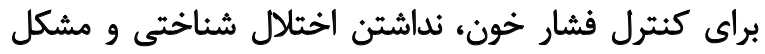

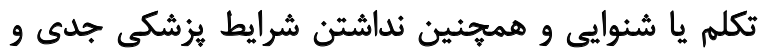

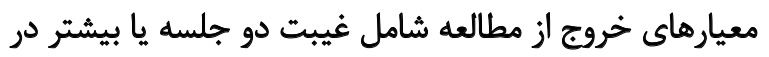

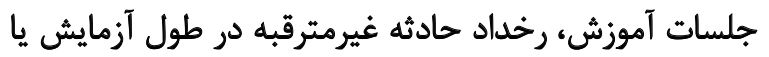

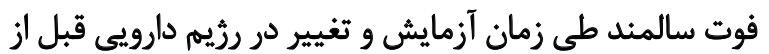
مطالعه مؤثر بر فشار خون بون آنمايث.

براى جمعآورى اطلاعات از فرم اطلاعات جمعيتشناختى لئى

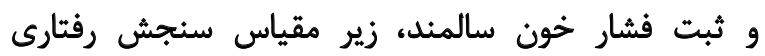

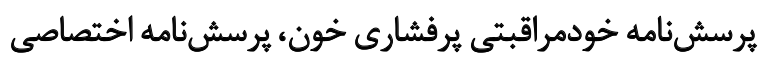

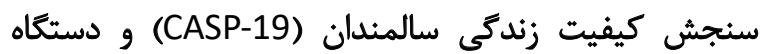

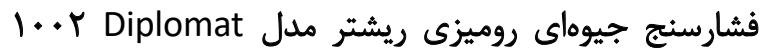

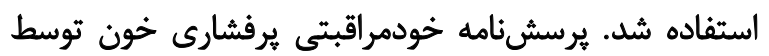

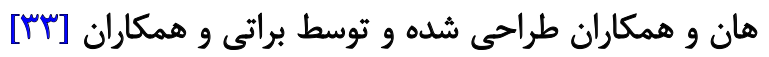
براى سالمندان ايرانى روانسنجى شده است وست براني

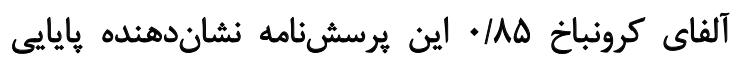

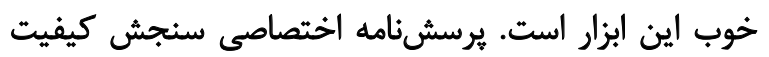
زندكى سالمندان (19-CASP) توسط هايدى و همكاران 
به ترتيب از آزمونهاى تى مستقل، من ويتنى و تى زوجى،

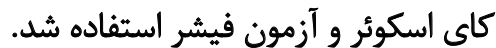

ياقتهها

در مطالعه حاضر، هشتاد نفر (با نسبت برابر در دو گروه)، با بالي ميانكين

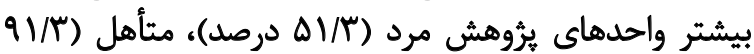

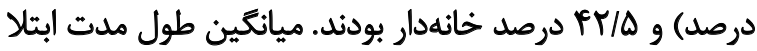

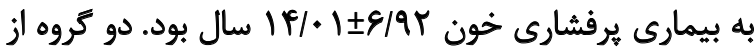

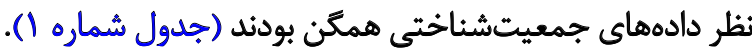

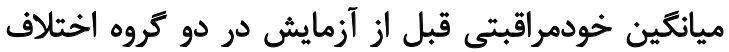

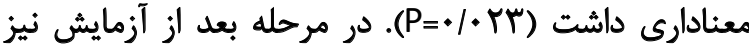

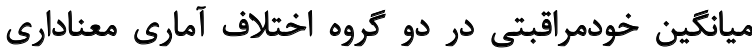

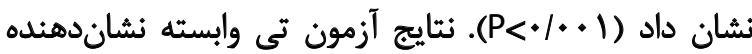

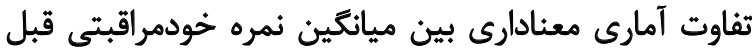

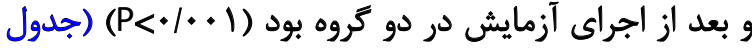

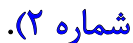

ميانگين كيفيت زندگى قبل از آزمايش در تروه اختلاف
يويكيرى عملكرد: در اين مرحله عملكرد بيماران به مدات

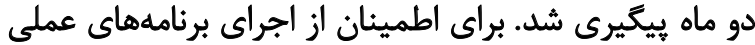

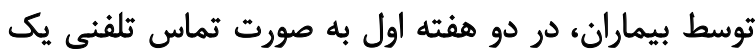

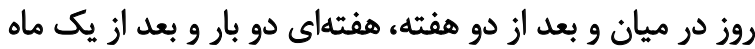

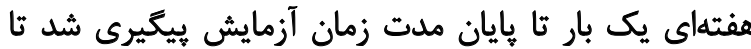

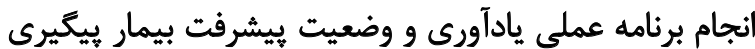

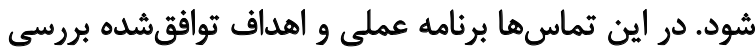

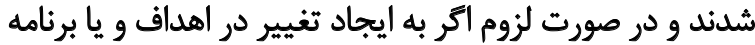

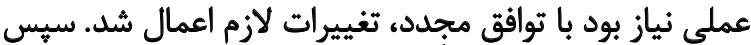

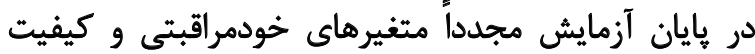

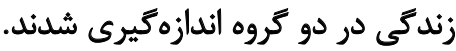

دادها يس از جمعآورى با استفاده از نرمافزار SPSS نسخه

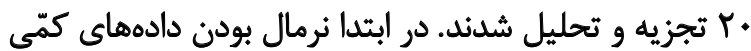

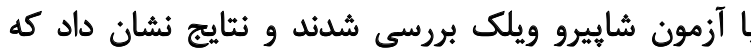

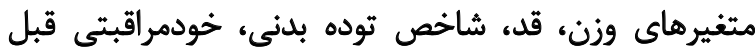

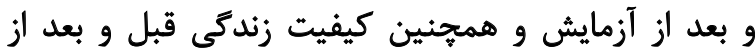

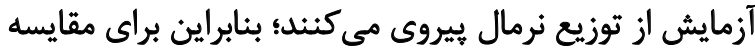

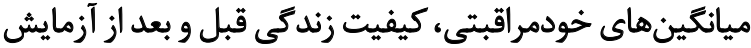

جدول ا. مقايسه ويركى هاى جمعيتشناختى در دو كروه آزمايش وكنترل

\begin{tabular}{|c|c|c|c|c|}
\hline \multirow{2}{*}{$\mathbf{P}$} & \multicolumn{2}{|c|}{ ميانكين \اتحراف معيار } & \multirow{2}{*}{\multicolumn{2}{|c|}{ متغير }} \\
\hline & كروه كنترل & يروه آززمايش & & \\
\hline$+/ \pi r_{*}^{*}$ & $A \Psi / \cdot Y \pm r / \cdot \Lambda$ & $\varepsilon_{\Delta} / / \Delta \pm r / V \Lambda$ & \multicolumn{2}{|c|}{ سن (سال) } \\
\hline . & TE/gY \pm T/RT & $r V / A T \pm r / \Delta r$ & \multicolumn{2}{|c|}{ شاخص توده بلنى } \\
\hline$+/ T \Delta F^{*}$ & $9 V / \Delta \cdot \pm \Psi N / \Delta$ & $V \cdot / / Y \pm \Delta F / / \varepsilon$ & \multicolumn{2}{|c|}{ ميزان فعاليت (ساعت در ماه) } \\
\hline.$(1 \times 9)^{*}$ & $N V \cdot \pm \cdot / Q 8$ & $N \Delta T^{w} \pm 1 / \mathrm{V}$ & \multicolumn{2}{|c|}{ ميزان خواب (ساعت در شبانه روز) } \\
\hline.$/ m^{*}$ & $1 F / 9 . \pm V / T V$ & $|r /| r \pm g / \Delta H$ & \multicolumn{2}{|c|}{ هلت ابثلال (عاه) } \\
\hline & \multicolumn{2}{|c|}{ تعداد (درصد) } & \multicolumn{2}{|c|}{ سطوح هتغير } \\
\hline . $/ 8 q q^{* * * * *}$ & $\begin{array}{l}r e(q .) \\
r(1 .)\end{array}$ & $\begin{array}{l}r V(q r / \Delta) \\
r(V / \Delta)\end{array}$ & فوت همسر هئل & وضعيت تأهل \\
\hline.$/\left.M\right|^{* * * *}$ & $\begin{array}{l}q(Y T / \Delta) \\
I \Delta(Y V / \Delta) \\
\Delta(I Y / \Delta) \\
I / T V / \Delta)\end{array}$ & $\begin{array}{l}g(\mid \Delta) \\
r \cdot(\Delta \cdot) \\
1 .(r \Delta) \\
r(1 .)\end{array}$ & زبيد دييلم ديسواد & ميزان تحصيلات \\
\hline$\cdot / \Delta \cdot r^{+\infty * *}$ & $\begin{array}{c}r)(\Delta T / \Delta) \\
19(R / \Delta) \\
*(\cdot)\end{array}$ & $\begin{array}{l}I V(F Y / \Delta) \\
Y Y(\Delta \Delta) \\
Y(Y / \Delta)\end{array}$ & بيشتر ازئ حد كثاف كفاف حد كفاف & وضعيت اقتصادى \\
\hline ./VAros* & $\begin{array}{l}1 \&\left(\varphi_{+}\right) \\
A\left(r_{0}\right) \\
1 \&\left(f_{0}\right)\end{array}$ & 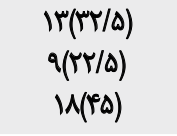 & خازئشسته & وضعيت شغلى \\
\hline$\circ 1+A V^{* 0 * * *}$ & $\begin{array}{l}r A(q \Delta) \\
r(\Delta)\end{array}$ & $\begin{array}{l}M(\Lambda \cdot) \\
\Lambda(T \cdot)\end{array}$ & كمنمك كميرب & 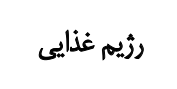 \\
\hline
\end{tabular}

ـ

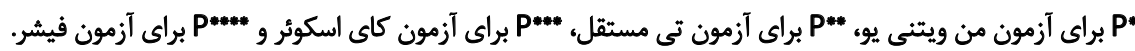


جدول Y. مقايسه ميانكين خودمراقبتي در دو كروه آزمايش و كنترل قبل و بعد از آزمايش

\begin{tabular}{|c|c|c|c|}
\hline \multirow{2}{*}{ آزمون تى مستقل } & \multicolumn{2}{|c|}{ ميانكَين \pm انحراف معيار } & \multirow{2}{*}{ متغيرها } \\
\hline & كنترل & آزمايش & \\
\hline $\begin{array}{c}t=r / H^{\prime} \\
d f=Y A \\
P=+/+r r\end{array}$ & $P T / V \Delta \pm \Delta / f e$ & $P E / E V \pm \Delta / A Y$ & خودمراقبثى قبل از آزمايش \\
\hline $\begin{array}{c}t=q / r+ \\
d f=v A \\
P<+/ *+1\end{array}$ & $P \cdot / 9 Y \pm T / N E$ & $\Delta . / \Omega^{\prime} \pm \pm / W$ & خودهراقبتى بعد از آزمايش \\
\hline & $\begin{array}{c}t=-v / \& 8 \\
d f=r q \\
P<+1 *+1\end{array}$ & $\begin{array}{c}t=N r \Delta \\
d f=r q \\
P<\cdot / \cdots 1\end{array}$ & نتيجه آزمون تى وابسته \\
\hline
\end{tabular}

il

جدول ب. مقايسه ميانكين كيفيت زندكى در دو كروه آزمايش و كنترل قبل و بعد از آزمايش

\begin{tabular}{|c|c|c|c|}
\hline \multirow{2}{*}{ آزمون تى مستقل } & \multicolumn{2}{|c|}{ ميانكين土|نحراف معيار } & \multirow{2}{*}{ متغيرها } \\
\hline & كتترل & أزمايشُ & \\
\hline $\begin{array}{c}t=r / e r \\
d f=V A \\
P<+1++1\end{array}$ & $r \Delta / \Delta \Delta \pm N M T$ & $r r / \& \Delta \pm N \cdot r$ & كيفيت زندىى قبل از آزمايش \\
\hline \multirow[t]{2}{*}{$\begin{array}{c}\mathrm{t}=\mathrm{N} / 9 \mathrm{q} \\
\mathrm{df}=\mathrm{VA} \\
\mathrm{P}<+1 * \cdot 1\end{array}$} & $\mathrm{rr} / \mathrm{qV} \pm \mathrm{V} / \mathrm{Iq}$ & $r q \cdot r \pm \& / V E$ & كيفيت زندكى بعد از آزمايش \\
\hline & $\begin{array}{c}t=-/ / q F r \\
d f=r q \\
P=* / r d r\end{array}$ & $\begin{array}{c}t=q / A r \\
d f=r q \\
P<+/ *+1\end{array}$ & نتيجه آزمون ثى وابسته \\
\hline
\end{tabular}

il

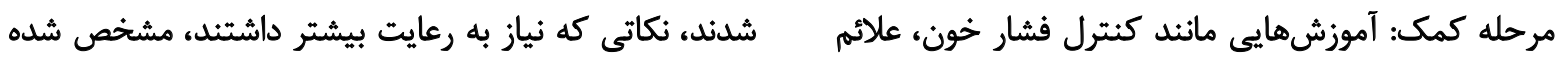

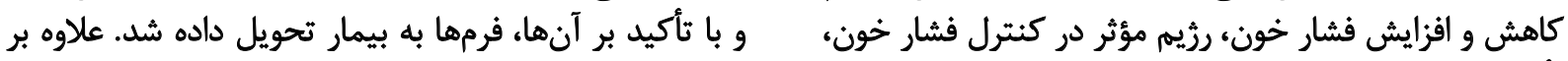

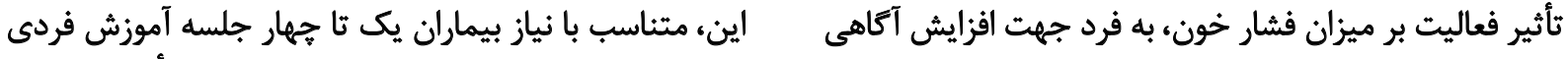

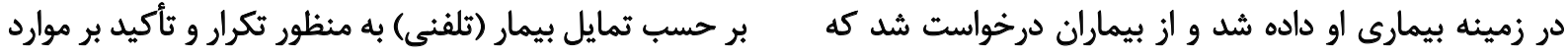

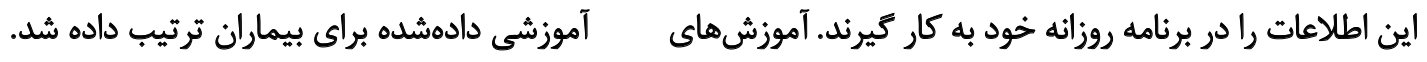

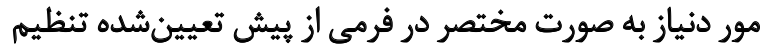

جدول P. مقايسه اختلاف ميانكين خودمراقبتى و كيفيت زندكى قبل و بعد از آزمايش در دو كروه موردمطالعه.

\begin{tabular}{|c|c|c|c|}
\hline \multirow{2}{*}{ تتيجه أزمون تى مستقل } & \multicolumn{2}{|c|}{ انحراف معيار_مياتئين } & \multirow{2}{*}{ هتغيرها } \\
\hline & كثترل & آزمايش & \\
\hline $\begin{array}{c}t=11 / / V \\
d f=V A \\
P<+/ \cdots 1\end{array}$ & $-r / A Y \pm T / M T$ & $P / T V \pm T / T V$ & اختلاف خودمراقبتى قبل و بعد از آزمايش \\
\hline $\begin{array}{c}t=V / r \Delta \\
d f=V A \\
P<\cdots / \cdots 1\end{array}$ & $-\cdot / \Delta V \pm r / N C$ & $\Delta / r \checkmark \pm \Psi / \uparrow \varepsilon$ & اختلاف كيفيت زندكى قبل و بعد از آزّمايش \\
\hline
\end{tabular}

L 
جهت سنجش تأثير توانمندسازي خود مراقبتى با استفاده از

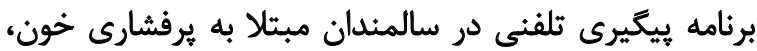

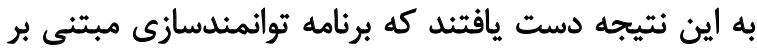

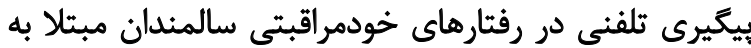

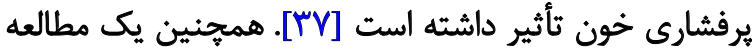

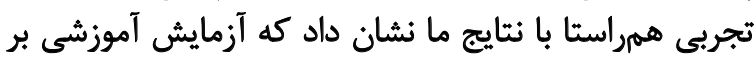

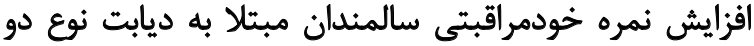
تأثير دارد [9].

اين سه مطالعه از نظر روش ارائه آموزش، محتواي ارائهشده،

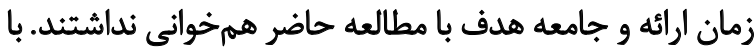

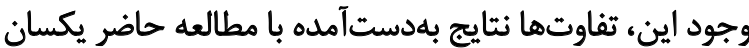

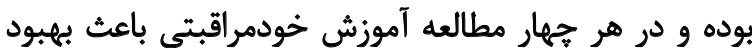
ميانكين نمره خودمراقبتى در جامعه تحت مطالعه شده است. فرايند بير شدن جمعيث با افزايش شيوع بيمارىهاى

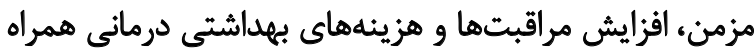

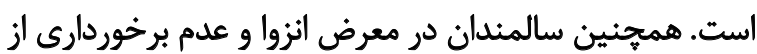

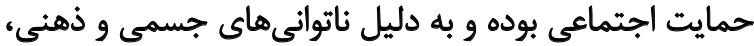

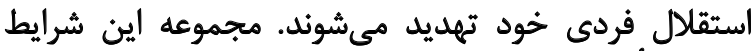

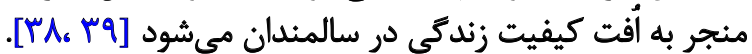
با توجه به اينكه كيفيت زندكى قبل از از آزمايش در دور

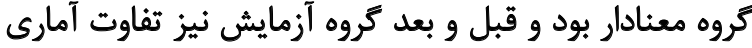

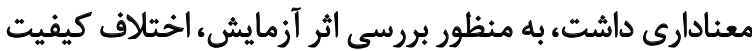

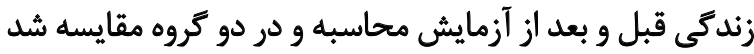

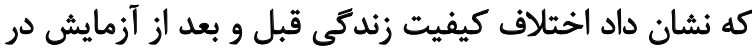

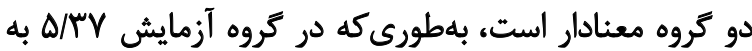

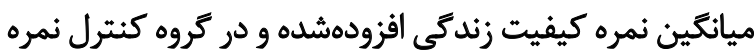
كيفيت زندكى

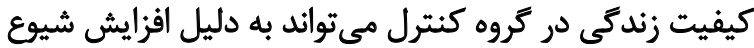

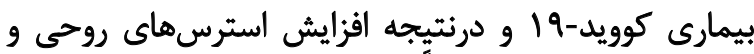

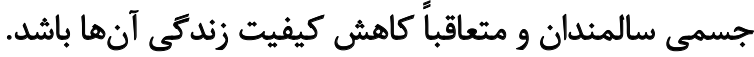

جوانوش و همكاران [11] در مطالعهاى با استفاده از مدل

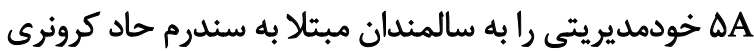

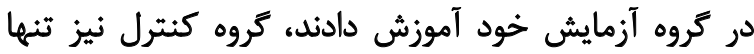

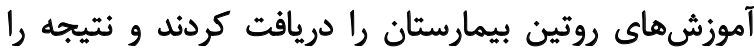

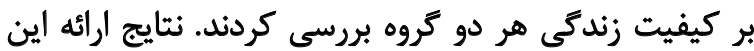

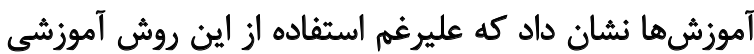

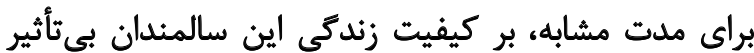

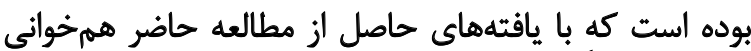

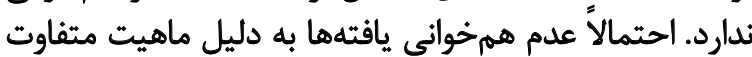

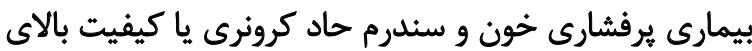

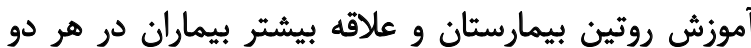

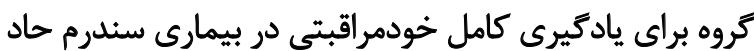

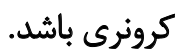

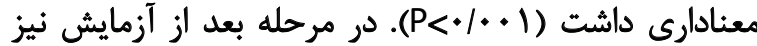

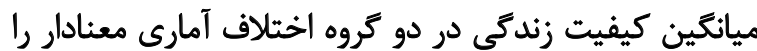

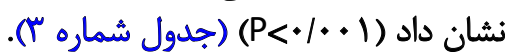
براي اطمينان از اثر آزمايش، آزمون اختلاف ميانكينهاى

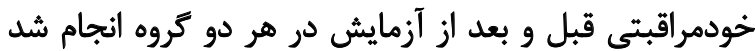

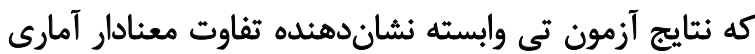

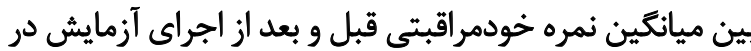

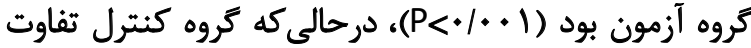

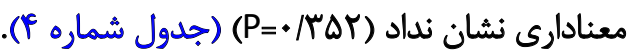

tras

مراقبت از خود، نقش ويثواى در يرفشارى خون سالمند دارد

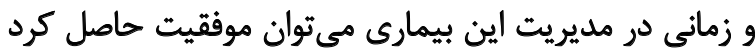

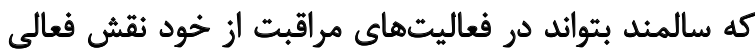

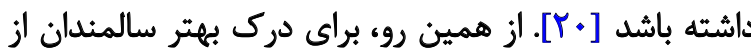

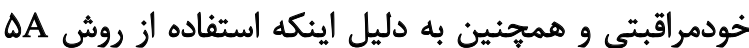

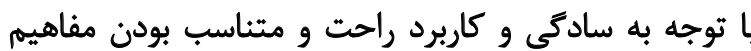

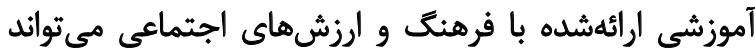

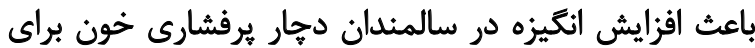

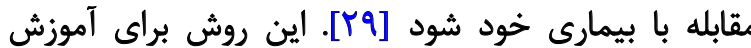

$$
\text { خودمراقبتى كارآي آمد است. }
$$

در مطالعه حاضر ميانكين نمره خودمراقبتى و كيفيت زندمكى

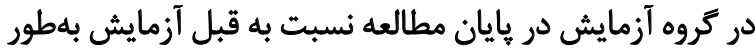
معنادارى افزايش يافت.

با توجه به اينكه خودمراقبتى قبل از آزمايش در دو ترو مروه

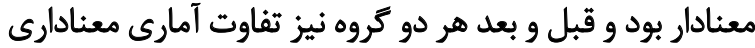

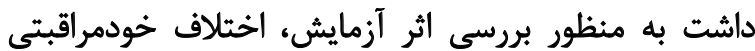

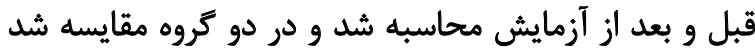

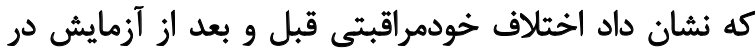

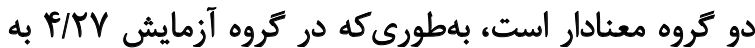

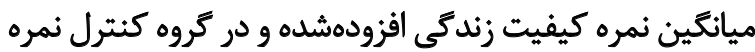

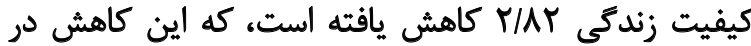

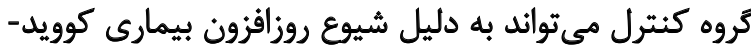

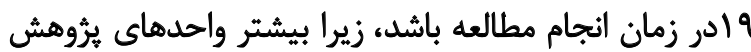

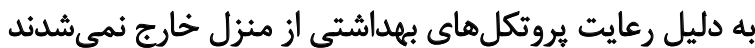

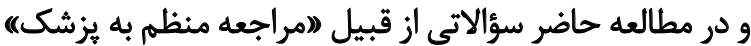

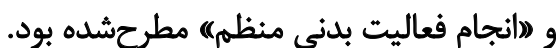

در مطالعهاى بيرامون اثربخشى آزمايش فعال سازى رفتارى

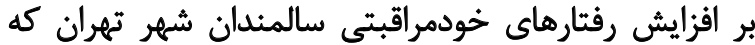

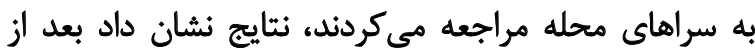

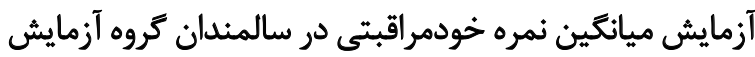
بهطور معنادارى افزايش يافته بود [عَّ]. در مطالعهاى ديكر 


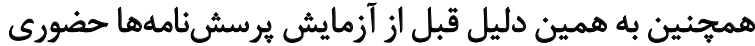
و بعد از آزمايش تلفنى تكميل شئ شد كه شايد اين آمائ امور بر صحت

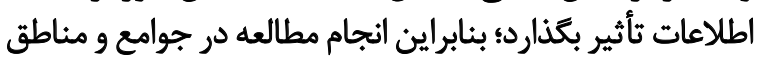

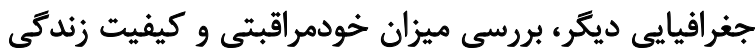

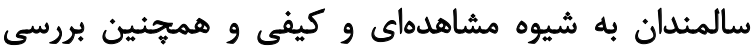
مجدد ئروهش يس از اتمام همهكيرى بيمارى كوريد بركشت شرايط عادى توصيه مى شود.

\section{نتيجه كَيرى نهايى}

هA نتايج مطالعه حاضر نشان داد كه آزمايش بر اساس مدل مدرل

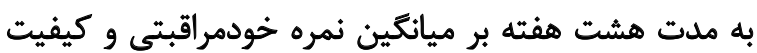

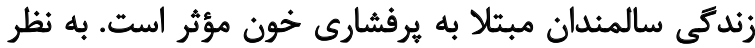

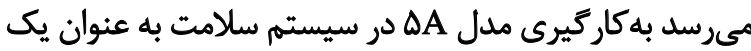

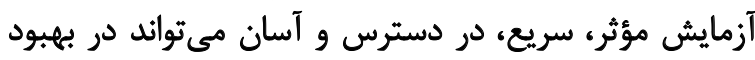

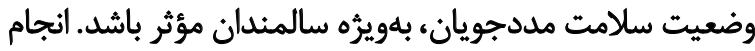

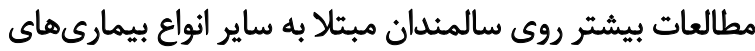

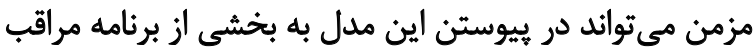

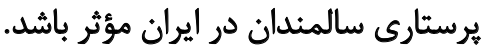

ملاحظات اخلاقي

\section{يبيروى أز اصول اخلاق بؤهش}

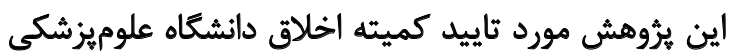

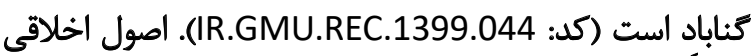

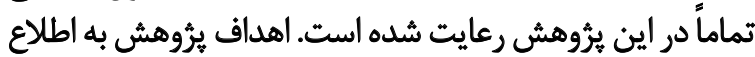

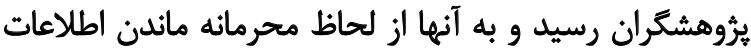

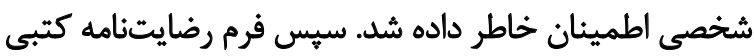
از شركت كنيدكان ترفته شد.

$$
\text { مامى مالى }
$$

اين مقاله بركرفته از ياياننامه كارشناسى ارشد نويسنده اول

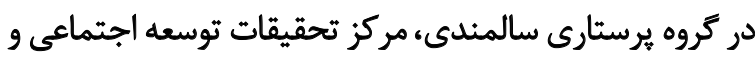

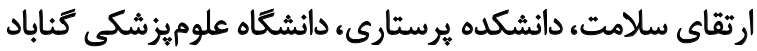

$$
\text { مشاركت نويسندكّان }
$$

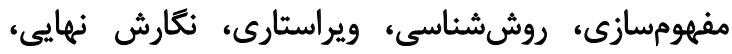

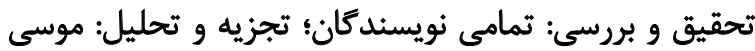

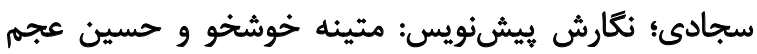

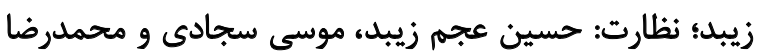

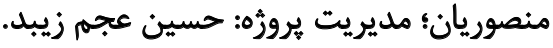

در يروهش حاضر از مدل هA براى آموزش سالمندان

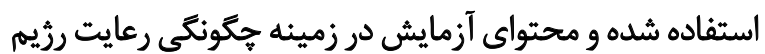

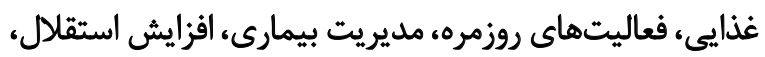

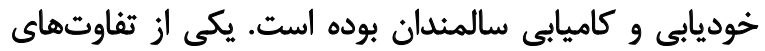

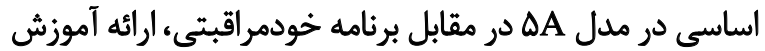

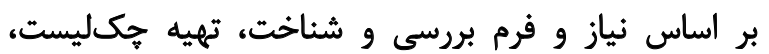

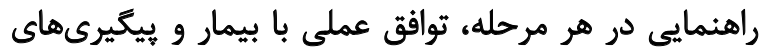

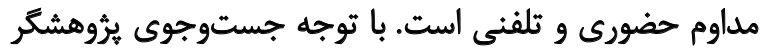

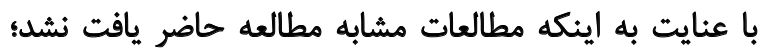

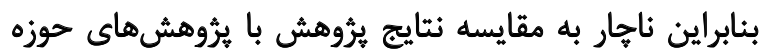
خودمراقبتى شده است.

يروهشكران در يك مطالعه تجربى با آموزش الكوى

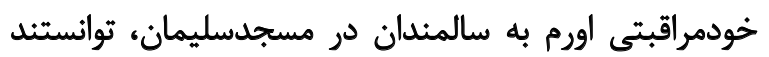

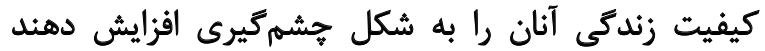

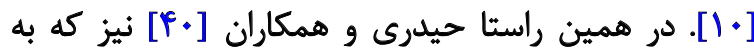

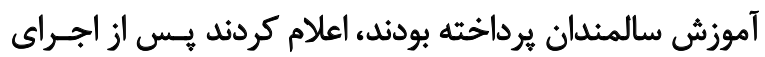

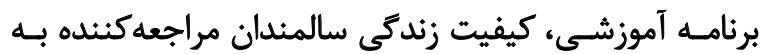

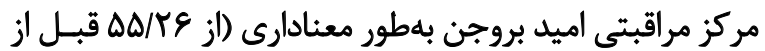

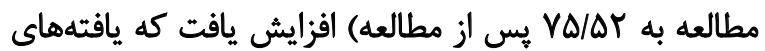

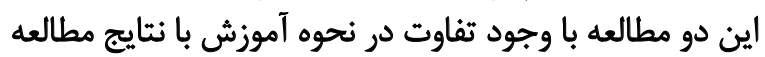
حاضر همخوانى داشت.

در تحقيقات انجامشده در كشورهاي توسعهيافته نيز نتايج

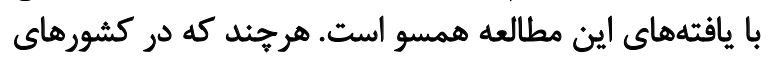

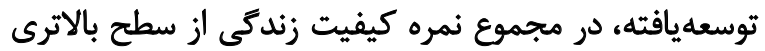

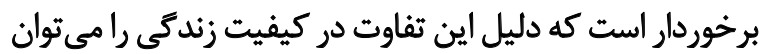

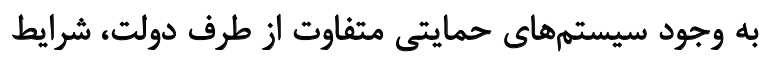

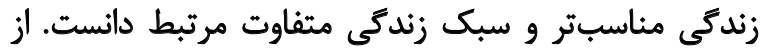

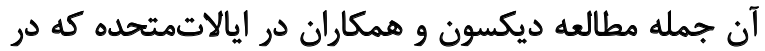

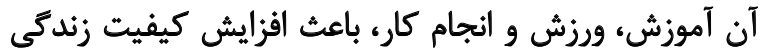

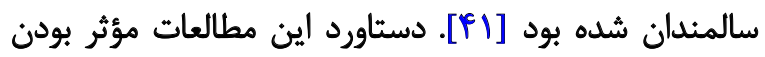

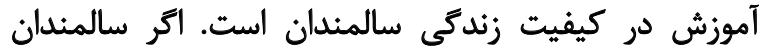

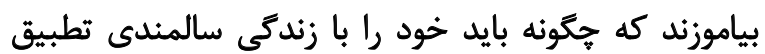

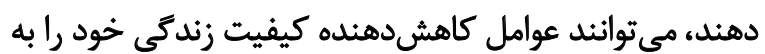
نحو مطلوبترى تعديل كنئد.

محدوديتهاى اين يروهش شامل موارد زير بود: بررسى في

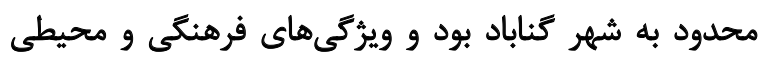

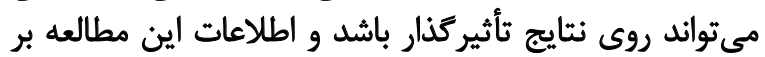

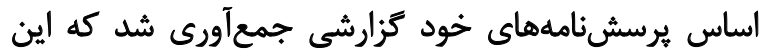

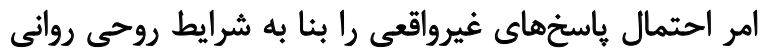

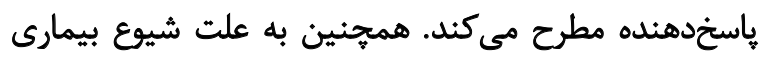

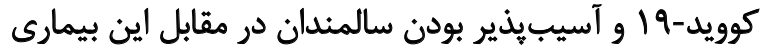

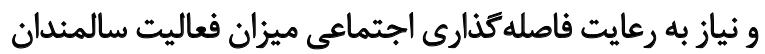

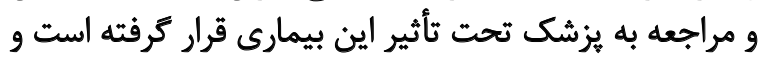




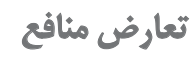

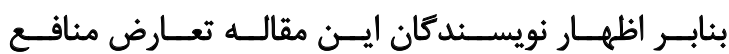

نـــــارد.

$$
\text { تشكر و قدورافي }
$$

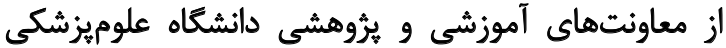

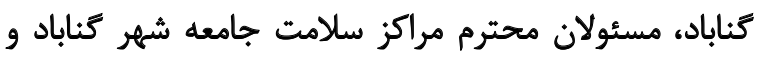

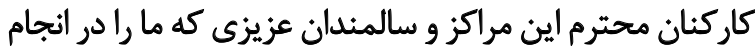

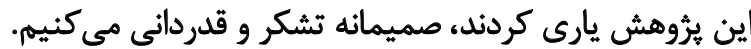




\section{References:}

[1] Taheri Tanjani P, Varmaghani M, Nazari N, Sharifi F, Fakhrzadeh H, Arzaghi SM, et al . [Health status of elderly people in East Azarbaijan: A cross-sectional study (Persian)]. Iranian Journal of Diabetes and Metabolism. 2017; 16(5):249-60. http://ijdld.tums. ac.ir/article-1-5420-en.html

[2] Hertog S. World population ageing 2017 [Internet]. 2017 [Updated 2017 October 5]. Available from: https:/ / www.un.org/development/desa/ageing/wp-content/uploads/sites/24/2017/05/ WPA-2017-Launch-to-the-IDOP-5-October-2017.pdf

[3] World Health Organization (WHO). World report on ageing and health. Geneva: WHO; 2015. https://books.google.com/books?i $\mathrm{d}=\mathrm{n} 180 \mathrm{DgAAQBAJ} \&$ printsec $=$ frontcover\&dq

[4] Statistical Center of Iran. Census 2016 - General results [Internet]. 2016 [Updated 2016]. Available from: https:/ / www.amar.org.ir/ english/Population-and-Housing-Censuses/Census-2016-General-Results

[5] Hidari H, Bolurchifard F, Yaghmaei F, Naseri N, Hamadzadeh S. [The effect of short-term aerobic exercise on the blood pressure in elderly clients with hypertension (Persian)]. Medical - Surgical Nursing Journal. 2014; 3(1):e87735. https:// sites.kowsarpub. $\mathrm{com} / \mathrm{msnj} /$ articles/87735.html

[6] Schneider RH, Alexander CN, Staggers F, Orme-Johnson DW, Rainforth M, Salerno JW, et al. A randomized controlled trial of stress reduction in African Americans treated for hypertension for over one year. American Journal of Hypertension. 2005; 18(1):8898. [DOI:10.1016/j.amjhyper.2004.08.027] [PMID] [PMCID]

[7] Cheraghi P, Mihandoost Yeganeh Z, Doosti Irani A, Sangestani M, Cheraghi Z, Khezeli M. [Study on the prevalence of hypertension and its associated factors in the elderly population (Persian)]. Journal of Geriatric Nursing. 2015; 1(3):73-86. http://ign.medilam.ac.ir/article-1-106-en.html

[8] Ahangari M, Kamali M, Arjmand Hesabi M. [The study of quality of life in the elderly with hypertension who are member of Tehran senile culture house clubs (Persian)]. Salmand: Iranian Journal of Ageing. 2008; 3(1):26-32. http://salmandj.uswr.ac.ir/ article-1-80-en.html

[9] Bagheri M, Bagheri M, Niknami Sh. [The effect of educational intervention on knowledge and self-care of elderly people with type 2 diabetes (Persian)]. Journal of Gerontology. 2018; 3(3):2130. [DOI:10.29252/joge.3.2.21]

[10] Baraz Sh, Rostami M, Farzianpor F, Rasekh A. [Effect of Orem self care model on ederies' quality of life in health care centers of Masjed Solaiman in 2007-2008 (Persian)]. Journal of Arak University of Medical Sciences. 2009; 12(2):51-9. http://jams.arakmu. ac.ir/article-1-119-en.html

[11] Javanvash Z, Mojdekanloo M, Rastaqhi S, Rad M. [The effect model-based self-management program 5A on quality of life of elderly patients with acute coronary syndrome Bojnourd Year 1395 (Persian)]. Journal of Sabzevar University of Medical Sciences. 2018; 25(1):75-82. http://jsums.medsab.ac.ir/article_1032. $\mathrm{html}$

[12] Hazavehei MM, Dashti S, Moeini B, Faradmal J, Shahrabadi R, Yazdi AH. [Factors related to self-care behaviors in hypertensive individuals based on Health Belief Model (Persian)]. Koomesh. 2015; 17(1):37-44. http://koomeshjournal.semums.ac.ir/article1-2154-en.html
[13] Hu H, Li G, Arao T. Prevalence rates of self-care behaviors and related factors in a rural hypertension population: A questionnaire survey. International Journal of Hypertension. 2013; 2013:526949. [DOI:10.1155/2013/526949] [PMID] [PMCID]

[14] Chodosh J, Morton SC, Mojica W, Maglione M, Suttorp MJ, Hilton L, et al. Meta-analysis: Chronic disease self-management programs for older adults. Annals of Internal Medicine. 2005; 143(6):427-38. [DOI:10.7326/0003-4819-143-6-200509200-00007] [PMID]

[15] Yang SO, Jeong GH, Kim SJ, Lee SH. Correlates of self-care behaviors among low-income elderly women with hypertension in South Korea. Journal of Obstetric, Gynecologic \& Neonatal Nursing. 2014; 43(1):97-106. [DOI:10.1111/15526909.12265] [PMID]

[16] Lee TW, Ko IS, Lee KJ. Health promotion behaviors and quality of life among community-dwelling elderly in Korea: A crosssectional survey. International Journal of Nursing Studies. 2006; 43(3):293-300. [DOI:10.1016/j.ijnurstu.2005.06.009] [PMID]

[17] Oraki M, Mehdizadeh A, Dortaj A. [The effectiveness of self-care empowerment training on life expectancy, happiness and quality of life of the elderly in Iranian elderly care centers in Dubai (Persian)]. Salmand: Iranian Journal of Ageing. 2019; 14(3):320-31. http:// salmandj.uswr.ac.ir/article-1-1663-en.html

[18] Hemmati Maslak Pak M, Hashemlo L. [Design and psychometric properties of a self-care questionnaire for the elderly (Persian)]. Salmand: Iranian Journal of Ageing. 2015; 10(3):12031. http:/ / salmandj.uswr.ac.ir/article-1-693-en.html

[19] Salehi L, Keikavoosi-Arani L. [Using the backman model in determining the dimensions of self-care and its factors affecting the elderly in Tehran city, Iran (Persian)]. The Horizon of Medical Sciences. 2020; 26(4):382-97. [DOI:10.32598/hms.26.4.3251.1]

[20] Masoudi R, Mohammadi E, Ahmadi F, Hasanpour-Dehkordi A. [The effect of self-care program education based on Orem's theory on mental aspect of quality of life in multiple sclerosis patients (Persian)]. Iran Journal of Nursing. 2009; 22(60):53-64. http:/ /ijn.iums.ac.ir/article-1-711-en.html

[21] Esmaeili R, Esmaeili M. [Quality of life in the elderly: A metasynthesis (Persian)]. Journal of Pizhūhish dar dīn va Salāmat. 2018; 4(2):105-16. [DOI:10.22037/jrrh.v4i2.18436]

[22] Darvishpoor Kakhki A, Abed Saeedi J, Delavar A, Saeed-OZakerin M. [Tools for measurement of health status and quality of life of elderly people (Persian)]. Research in Medicine. 2010; 33(3):162-73. http:// pejouhesh.sbmu.ac.ir/article-1-663-en. html

[23] Eyni S, Hosseini SA, Ebadi M, Mohammadzadeh M. [Causal modeling for the quality of life based on anxiety sensitivity through the mediating role of experiential avoidance in the elderly (Persian)]. Aging Psychology. 2020; 6(1):65-77. https:// jap.razi.ac.ir/article_1474.htm

[24] Teasdale MR. Quality of life and mission. Missiology: An International Review. 2016; 44(3):269-80. [DOI:10.1177/0091829616645135]

[25] Sotodeh Asl N, Neshat Dost H, Kalantery M, Talebi H, Khosravi AR. [Comparison of the effectiveness of cognitive behavioral therapy and medication on the quality of life in the patients with essential hypertension (Persian)]. Koomesh. 2010; 11(4):294-301. http://koomeshjournal.semums.ac.ir/article1-816-en.html 
[26] Warren-Findlow J, Seymour RB, Brunner Huber LR. The association between self-efficacy and hypertension self-care activities among African American adults. Journal of Community Health. 2012; 37(1):15-24. [DOI:10.1007/s10900-011-9410-6] [PMID] [PMCID]

[27] Moattari M, Ghobadi A, Beigi P, Pishdad GR. Impact of selfmanagement on metabolic control indicators of diabetes patients. Journal of Diabetes \& Metabolic Disorders. 2012; 11:6. [DOI:10.1186/2251-6581-11-6] [PMID] [PMCID]

[28] Kalhor M, Fathi M, Ghaderi B, Roshani D, Ozairi Sh, Rashidi $\mathrm{K}$. [Effect of five a model self-management on quality of life in patients with breast cancer (Persian)]. Avicenna Journal of Nursing and Midwifery Care. 2019; 27(4):269-80. [DOI:10.30699/ ajnmc.27.4.269]

[29] Moradi M, Nasiri M, Haji Ahmadi M, Gahanshahi M. [The effect of self-management program based on $5 \mathrm{~A}$ model on coping strategies in the elderly men patients with hypertension (Persian)]. Journal of Geriatric Nursing. 2017; 4(1):32-47. http://jgn. medilam.ac.ir/article-1-238-en.html

[30] Sadeghigolafshanl M, Rejeh N, Heravi-Karimooi M, Tadrisi $\mathrm{SD}$. [The effect of model-based self-management program $5 \mathrm{~A}$ on self-efficacy of elderly patients with diabetes (Persian)]. Journal of Diabetes Nursing. 2020; 8(1):1002-10. http://jdn.zbmu.ac.ir/ article-1-391-en.html

[31] Seyam Sh, Hidarnia AR, Tavafian SS. [Self-care education through coping style for patients after heart surgery (Persian)]. Journal of Isfahan Medical School. 2011; 29(135):433-44. http:/ / jims.mui.ac.ir/index.php/jims/article/view/1125

[32] Lissolo S, Cavallo E, Ronchetto F. [The problem of the chronic recurrent pain among older in-patients (Italian)]. Minerva Medica. 2006; 97(2):205-15. [PMID]

[33] Barati F, Sadeghmoghadam L, Sajjadi M, Nazari Sh, Bahri N. Validation of the Persian version of self-care tools for hypertension among older adults. Medicinski Glasnik. 2019; 16(2):338-43. [DOI:10.17392/1026-19] [PMID]

[34] Heravi-Karimooi M, Rejeh N, Garshasbi E, Nikkhah M, Montazeri A. [A validation study of the Persian version of older people's quality of life questionnaire (CASP-19) (Persian)]. Payesh. 2016; 15(4):432-42. http:/ / payeshjournal.ir/article-1-166-fa.html

[35] Fakhri A, Morshedi H, Mohammadi Zeidi I. [Effectiveness of theory based education on medication adherence in older adults with hypertension (Persian)]. Jundishapur Scientific Medical Journal. 2017; 16(2):161-74. [DOI:10.22118/JSMJ.2017.49241]

[36] Naji Esfahani F, Seirafi MR, Kraskian Mujembari A. [The effectiveness of behavioral activation intervention on increasing selfcare behaviors and life expectancy in the elderly (Persian)]. Aging Psychology. 2020; 6(2):93-105. [DOI:10.22126/JAP.2020.5344.1427]

[37] Behzad Y, Bastani F, Haghani H. [Effect of empowerment program with the telephone follow-up (tele-nursing) on self - efficacy in self-care behaviors in hypertensive older adults (Persian)]. Nursing and Midwifery Journal. 2016; 13(11):1004-15. http:// unmf.umsu.ac.ir/article-1-2422-en.html

[38] Vahdani Nia MS, Goshtasebi A, Montazeri A, Maftoon F. [Health-related quality of life in an elderly population in Iran A population-based study (Persian)]. Payesh. 2005; 4(2):113-20. http:/ / payeshjournal.ir/article-1-778-fa.html

[39] Wilcox S, Sharkey JR, Mathews AE, Laditka JN, Laditka SB, Logsdon RG, et al. Perceptions and beliefs about the role of physical activity and nutrition on brain health in older adults. The Gerontologist. 2009; 49(S1):S61-71. [DOI:10.1093/geront/gnp078] [PMID]
[40] Heidari M, Shahbazi S. [Effect of self-care training program on quality of life of elders (Persian)]. Iran Journal of Nursing. 2012; 25(75):1-8. http://ijn.iums.ac.ir/article-1-1229-en.html

[41] Dickson VV, Howe A, Deal J, McCarthy MM. The relationship of work, self-care, and quality of life in a sample of older working adults with cardiovascular disease. Heart \& Lung. 2012; 41(1):514. [DOI:10.1016/j.hrtlng.2011.09.012] [PMID] 
This Page Intentionally Left Blank 